Published in final edited form as:

J Am Chem Soc. 2013 October 30; 135(43): . doi:10.1021/ja409056b.

\title{
Correction to Real-Time Observation of Multiple-Protein Complex Formation with Single-Molecule FRET
}

\author{
Wooli Bae, Mal-Gi Choi, Changbong Hyeon, ${ }^{\text {Y Yeon-Kyun Shin, }}$ and Tae-Young Yoon ${ }^{*}$ \\ [J. Am. Chem. Soc., 2013, 135, 10254-10257] \\ Page 10254. The author's name should read Wooli Bae. \\ ja404276g
}

